\title{
Acute otitis media- an update
}

\begin{abstract}
Objective: To review the literature about Acute otitis media (AOM).

Introduction: AOM is a common condition with an incidence of 10.8/ 100 people per year; it is more common in pediatric age group and tends to occur more in male patients. It is considered the main cause of prescribing antibiotics in pediatrics. Physicians tend to over diagnose this disease leading to an unnecessary antibiotics prescription which exposes the child to an unwanted side effects mainly diarrhea. USA spends \$2-3.5billion per year for the treatment of AOM. Taking these points in our mind, it is important to know how to accurately diagnose this condition and how to treat it and what is the pathophysiology that underlies that disease, all of which are discussed in details in this review article.
\end{abstract}

Methodology: a comprehensive review of the literature about AOM.

Results: AOM is a result of different host, environmental, microbiological and genetic factors. It is highly related to upper respiratory infection and the disease is thought to be viral in origin with bacterial superinfection happens later on. Respiratory syncytial virus (RSV) has the best link with AOM. Streptococcus pneumoniae, Haemophilus influenzae and Moraxella catarrhalis are still the most important pathogens. It is a symptomatic disease; Ear pain is the most important symptom, and bulging of the tympanic membrane is the most important sign that can lead to diagnosis. The American Academy of Pediatrics has put certain guidelines for the treatment of AOM. Prevetive measures play an important role in decreasing of its incidence.

Conclusion: AOM has an impact on the child, family, and the community as a whole, in term of health and costs.

Keywords: acute otitis media, spectrum, pathogenesis, otorrhea
Volume 8 Issue 4 - 2017

\author{
Hassan Haidar, ${ }^{1,2}$ Ma'in Al Shawabkeh,' \\ Aisha Larem, ${ }^{1,2}$ Zahraa Albu Mahmood, ${ }^{3}$ Ali \\ Alsaadi, ${ }^{1,2}$ Abdulsalam Alqahtani ${ }^{1,2}$ \\ 'Otolaryngology Department Hamad Medical Corporation, \\ Qatar \\ ${ }^{2}$ Otolaryngology Department Weil Cornell Medical College, \\ Qatar \\ ${ }^{3}$ Family Medicine Department Hamad Medical Corporation, \\ Qatar
}

Correspondence: Hassan Haidar ENT consultant Hamad Medical Corporation Doha, Qatar, Email Hahmad@hamad.qa

Received: June 17,2017 | Published: August 31, 2017
Abbreviations: AOM, acute otitis media; OME, otitis media with effusion; TM, tympanic membrane; RSV, respiratory syncytial virus; MRI, magnetic resonance imaging; URTI, upper respiratory infection

\section{Introduction}

Otitis media $(\mathrm{OM})$ has a wide spectrum of diseases that include acute otitis media (AOM), recurrent otitis media (rOM), otitis media with effusion (OME), chronic otitis media with effusion and chronic otitis media, ${ }^{1,2,3}$ AOM can be defined as a rapid onset of fever and otalgia, ${ }^{1,2,4}$ if the child has $\geq 3$ episodes of AOM in the preceding 6 months, or $\geq 4$ episodes in the preceding 12 months then he is considered to have recurrent $\mathrm{OM},{ }^{1,5,6}$ presence of fluid in the middle ear with absence of signs and symptoms is defined as otitis media with effusion (OME), and if the fluid persisted more than 3months then it is considered as chronic otitis media with effusion, ${ }^{1}$ chronic suppurative otitis is defined as discharge with tympanic membrane (TM) perforation for more than 3months. ${ }^{1}$ Patients who have more than 6 episodes in the first 6years of life are said to be otitis prone. ${ }^{7}$ This review article will scope more on AOM.

\section{Definitions}

American Academy put certain guidelines to define AOM; it includes severe or moderate bulging of the TM, new-onset otorrhoea without otitis externa being present, or mildly bulging TM associated with recent ( $<48$ hours) otalgia and intense TM Erythema. ${ }^{5,7,8}$ AOM can be divided into severe and non severe, complicated and non complicated, ${ }^{8}$ if the patient has moderate to severe otalgia with temperature $(\mathrm{T}) \geq 39^{\circ} \mathrm{C}$, then it is said to have severe $\mathrm{AOM},{ }^{5,8}$ whereas mild otalgia with $\mathrm{T}$ below 39 is enough to categorize him in the nonsevere group, ${ }^{5,8}$ uncomplicated AOM is the one without otorrhea. ${ }^{5}$

\section{Epidemiology}

AOM is considered the most common inflammatory disease, $, 7,910$ and the most common indication of prescribing antimicrobial in children., ${ }^{2,111,12}$ US spends \$2-3.5billion per year for treatment of this condition. ${ }^{2,13}$ In general it is a disease of childhood, that has a peak incidence of 6-11 months, ${ }^{1,8} 59 \%$ of children will have one episode of AOM by age of $2^{1}$ and $30 \%$ will have 2 or more episodes, ${ }^{1}$ males have a higher incidence of AOM and Rom. ${ }^{1,8}$ It has an incidence rate of $10.8 / 100$ people per year. ${ }^{14}$

\section{Pathogenesis}

AOM has multiple interactive host, environmental, microbiological and genetic factors that interplay with each other to make the patient more susceptible to have AOM. ${ }^{10,15,12}$

AOM is related to upper respiratory infection (URTI); in $94 \%$ of patient URTI proceeds $A O M,{ }^{1,7}$ one in three children will develop AOM after URTI. ${ }^{12}$ URTI causes nasopharyngitis which affects the Eustachian tube (ET), as a result negative pressure is created and that causes suction of the mucus of the nasopharynx inside the middle ear; that explains why aspirate of AOM can show bacteria like Streptococcus pneumoniae, Haemophilus influenzae and Moraxella catarrhalis, ${ }^{2,8}$ which are commensal to nasopharynx. ${ }^{16}$ The initial course of the disease is thought to be viral in origin with bacterial superinfection occurs after that, ${ }^{2,12}$ many viruses have been implemented as a causative agents which are found in $90 \%$ of AOM cases, like rhinovirus, coronavirus, enterovirus, respiratory syncytial virus (RSV), parainfluenza type 1,2 or 3, influenza A or $\mathrm{B}$, and adenovirus. ${ }^{1,2,7,14,17}$ Among all of these RSV is believed to have the best association with AOM. ${ }^{12}$ These viruses are thought to increase the adherence of the bacteria to the epithelium and by this 
enhances bacterial colonization, ${ }^{12,17}$ however, there is rising evidence that viruses can cause AOM without bacterial superinfection in 10$15 \%$ of the cases, a point that should be kept in mind while treating AOM with antibiotics. ${ }^{12,17}$

Genetics play an important role in the susceptibility of an individual to AOM. ${ }^{1,2,8,12,15}$ Native American Maori or Australian Aborigine populations have more prevalence of OM as compared to white population. ${ }^{1}$ In one cohort study showed that heritability is found in $38.5 \%$ in $\mathrm{rOM}^{15}$ Some investigators have linked this susceptibility to poor ET function. ${ }^{2}$ Specific polymorphisms of cytokines have been correlated to the susceptibility of an individual to OM and to the severity of the disease as well:;0,12 IL-1s, IL-6 and TNF $\alpha$ have been found in nasopharyngeal secretions during URTI and increased levels of IL-1s is linked to the transition to AOM. ${ }^{12}$ TNF $\alpha$ 308 polymorphisms are associated with AOM that follows URTI. In addition some recurrent $\mathrm{AOM}$ patients found to have $\mathrm{TNF} \alpha-308$ and IL-6-174 Polymorphisms. ${ }^{12}$ Genes for Fcg-receptor IIa (FcgRIIa), IL-10, CD14, toll-like receptor4 (TLR 4), surfactant, and interferon gamma (IFNg) are believed to be responsible for OM risk. ${ }^{15}$

The importance of other environmental factors cannot be underestimated in the pathogenesis of AOM; Paternal smoking is one of OM risk factors; ${ }^{1,3,8,10,14}$ it causes mucosal inflammation, goblet hyperplasia and an increase in the production of mucus, by this it impairs mucosal immunity and increases bacterial colonization by enhancing bacterial binding to respiratory epithelium. ${ }^{1,10}$ Children of mothers who smoked during pregnancy and stopped after delivery still have higher incidence of AOM ${ }^{10}$ Children with bottle feeding is another well known risk factor, ${ }^{1,3,8,10}$ it is believed that passive immunity through the breast milk gives some protection, ${ }^{1}$ bottle-fed infants have higher nontypeable Haemophilus influenzae prevalence and lower immunoglobulin G antibodies. ${ }^{8}$ Some studies found a role of Gastro esophageal reflux (GERD) disease, ${ }^{1,18}$ GERD is found in $62.9 \%$ of patient with rOM. In fact pepsin/pepsinogen can be found in the middle ear in $85.3 \%$ of OM patients, a finding that can be explained by the reflux; however a cause and effect relationship cannot be established. ${ }^{18}$ Certain vitamin and dietary deficiencies can be found in cases of AOM and rOM; like vitamin A, zinc, EPA (an omega 3 fatty acid) and selenium deficiencies. ${ }^{13}$ There are other risks factors that are related to AOM, like younger age, ${ }^{1,14}$ attendance to day care centers, ${ }^{3,8,10}$ prematurity, ${ }^{8}$ increased number of siblings, dummy use,,${ }^{1,8}$ Autumn and winter seasons, ${ }^{1}$ craniofacial anomaly, ${ }^{14}$ asthma ${ }^{3}$ and atop ${ }^{3,14}$ (Table 1).

Table I The risk factors of acute otitis media

\begin{tabular}{l}
\hline Risk Factors for AOM \\
\hline Young Age \\
\hline Day Care Attendance \\
\hline Exposure to Smoking \\
\hline Lack of Breast Feeding \\
\hline Supine Feeding Position \\
\hline Use of Pacifiers \\
\hline Family History of Recurrent AOM \\
\hline Craniofacial Abnormalities \\
\hline Immune Deficiency \\
\hline Gastroesophageal Reflux \\
\hline
\end{tabular}

\section{Microbiology}

AOM is usually a complication of an acute viral upper respiratory infection. Numerous large studies have documented the bacterial pathogens associated with the diagnosis of AOM.
Streptococcus pneumoniae, Haemophilus influenzaeand Moraxella catarrhalis are the most common pathogens. ${ }^{3,19,20}$ Streptococcus pneumonia is the most common and found in $35-45 \%,{ }^{3,20}$ patients infected with this microorganism tend to have more severe disease.,16 The serotypes responsible have been changed after the introduction of pneumococcal 7-valent conjugate vaccine (PCV7). ${ }^{3}$ Haemophilus influenza has become more common after PCV 7 vaccine. ${ }^{3}$ It is related to bilateral disease, recurrent cases, and conjunctivitis with the so called otitis conjunctivitis syndrome. ${ }^{3,16,19}$ Moraxella catarrhalis found in $10-20 \%{ }^{19}$ it is found in the first episode and mixed infection and $100 \%$ are beta lactamase producers. ${ }^{19}$ All of the above pathogens have the ability to form biofilms, ${ }^{16}$ which are thought responsible for the resistance to therapy. ${ }^{16}$

Group A Streptococcus, Group B Streptococcus, Staphylococcus aureus and gram-negative bacilli are other bacteria that can be encountered in approximately $15 \%$ of cases. ${ }^{3}$ First episode of bacterial infection with invasive strains (like invasive strains of Streptococcus pneumonia) can lead to mucosal changes which make the ear more susceptible to acquire the disease by less virulent strains. ${ }^{21}$

Approximately $5 \%$ of ears have multiple pathogens. No bacterial pathogen is identified by tympanocentesis in approximately $20-30 \%$ of ears with clinical AOM.

A few studies have also sought evidence of viral infection. Respiratory viruses including RSV, rhinovirus, adenovirus, influenza virus, parainfluenza virus, and CMV are isolated from approximately $20 \%$ of middle ear samples cultured. Overall, evidence of viral infection is detected in almost $50 \%$ of children with AOM, half of whom have bacterial/viral co-infections.

\section{In cases of simultaneous infection with patho}

genic viruses and bacteria, antibiotic responsiveness appears to be inferior to that of children with only bacterial infection, and microbiologic failure can occur despite clear in vitro sensitivity to the antibiotic used. Consequently, a second course of amoxicillin can effectively clear bacteria after the immune system has cleared the viral infection.

\section{Clinical manifestation}

Patients can present with otalgia;,2,7,8 ear pulling ${ }^{7,8}$ and reduced hearing, ${ }^{8}$ otalgia is considered the most important symptom as it has the highest positive predictive value but it is not reliable by its $\mathrm{own}^{2,4,7,11}$ but some patients may not have earache. ${ }^{4,11}$ Ear pulling is not specific as only $10 \%$ of ear pullers will have AOM. ${ }^{2}$ These acute symptoms tend to resolve within 4 days in $67 \%$ of cases. ${ }^{1}$ Predictive measures for $\mathrm{AOM}$ to convert to $\mathrm{rOM}$ in young children remain poor. ${ }^{22}$

\section{Diagnosis}

Clinical signs play an important role in diagnosis AOM as this disease occurs mainly in non-verbal children ${ }^{1}$. In fact, AOM diagnosis cannot be made without clinical signs of middle ear effusion. ${ }^{5}$

Diagnostic criteria: Acute otitis media (AOM) is defined as the combination of middle ear effusion (MEE), signs of inflammation such as:
i. Purulence
ii. Erythema
iii. Or a bulging tympanic membrane, and
iv. Symptoms of fever, otalgia, or irritability in young children. 
Diagnostic techniques: Basic question facing a clinician evaluating a patient's ears is whether or not MEE is present. If the presence or absence of MEE is less than clear, all available techniques should be used, including otoscopy, pneumatic otoscopy, and tympanometry.

Otoscopy: The most valuable technique for demonstrating the presence or absence of middle ear disease is the adequate visualization of normal landmarks. When the diagnosis of AOM is being considered, obstructing cerumen should be removed. The presence or absence of a light reflex is probably not a useful sign, and the tympanic membrane of a febrile or screaming child will sometimes appear reddened, even in the absence of middle ear disease. Injection of the TM alone has $7 \%$ positive predictive value (PPV) $;^{7}$ bulging of the TM is the single most important sign in diagnosing AOM with high $\mathrm{PPV}^{2,7,8,23}$ and its PPV is increased if combined with discoloration of TM and reduced mobility, ${ }^{4,23}$ but poorly mobile, opacified, bulging TM is found only in $19 \%$ of cases. ${ }^{4}$

Even under ideal conditions, simple otoscopy has a sensitivity and specificity of only $74 \%$ and $60 \%$, respectively for patients with middle ear effusion. Middle ear fluid can be present even with normal landmarks. This highlights the importance of including pneumatic otoscopy and/or tympanometry in the assessment of the middle ear.

Pneumatic otoscopy: Brisk movement of the tympanic membrane with slight application of pressure is normal. If the tympanic membrane does not move perceptibly with application of slight positive or negative pressure, a middle ear effusion is likely. Pneumatic otoscopy is an important tool, ${ }^{1,3,7,11}$, it has $90 \%$ sensitivity and $80 \%$ specificity 5,11. The position of the TM is the most important sign to distinguish between AOM and OME. ${ }^{5,23}$ Pneumatic otoscopy in general needs skills, good illumination and cerumen removal in order to accurately diagnose the case ${ }^{4,11}$. The major weakness of pneumatic otoscopy is the occasional difficulty of obtaining a good seal, especially in infants and in uncooperative children.

Tympanometry: Tympanometry is a tool that can be utilized in diagnosis especially in uncooperative children but it is not a replacement for physical examination. ${ }^{7,11}$ It has a $90 \%$ predictive value, ${ }^{11}$ it helps in detecting middle ear fluid, its use in primary care has led to decrease the over diagnosis of AOM by $30 \% .{ }^{11}$

Tympanocentesis: Tympanocentesis is considered the gold standard in diagnosing AOM, but it is utilized only in certain cases like failure of second line therapy to help in culture the aspirate to direct antibiotic therapy. ${ }^{4,7}$

Imaging studies: Imaging studies like Magnetic resonance imaging (MRI) and computed imaging study (CT) can be used if complications are suspected. ${ }^{7}$ Distinguishing between AOM and OME is essential as OME does not require antibiotic therapy, ${ }^{4}$ Otitis externa, myringitis and exacerbation of chronic otitis media are in the list of differential diagnosis of $\mathrm{AOM}^{?}$

\section{Complications}

Complications can occur after AOM.,.$^{3,19} \mathrm{TM}$ perforation happens in $7 \%$ of the cases and that tends to heal in $98 \%$ of cases in the first $1-2$ weeks, ${ }^{7}$ mastoiditis occurs in $1.11000,{ }^{7}$ bezold's abscess, citelli's abscess, luc's abscess can complicate mastoditis, ${ }^{7}$ facial palsy happens in $0.5-0.005 \%$ through a dehiscent facial canal, ${ }^{7}$ labyrinthitis and apical petrositis are other complications that can happen, ${ }^{3,7}$ Gradenigo's syndrome is another name for apical petrositis; ${ }^{7}$ it can present with the classical clinical trial of retrobulbar facial pain, discharging ear and abducens nerve palsy. ${ }^{7}$ Intracranial complications can follow AOM ${ }^{7}$ meningitis occurs in 0.42 per 100,$000 ;^{7}$ the pneumococcal is the most important pathogen linked to this complication. ${ }^{7}$ Brain abscess, lateral sinus thrombosis and otitic hydrocephalus are well know intracranial complications of $\mathrm{AOM}^{3,7,19}$ (Table 2)

Table 2 Acute Otitis Media complications

\begin{tabular}{ll}
\hline Extra-Cranial & Intra-Cranial \\
\hline Matoditis in I:I0000 & Meningitis in 0.24 per 100000 \\
\hline Bezold's Abscess & \\
\hline Citelli's Abscess & \\
\hline Luc's Abscess & Brain Abscess \\
\hline Facial palsy in 0.005\%-0.5\% & Lateral Sinus Thrombosis \\
\hline Labyrinthitis & Otitic Hydrocephalus \\
\hline Petrositis (Gradenigo's Syndrome)
\end{tabular}

\section{Management}

As stated before, AOM is the most common indication of prescribing antimicrobial in children, $2,7,11,12$ bearing this fact in mind it is important to know when to prescribe antibiotics. In $199090 \%$ of cases in USA, Australia, New Zealand, England and Wales were treated by antibiotics, ${ }^{23}$ this percentage was only $31 \%$ in Netherlands..$^{23}$ Diarrhea is the most common side effect encountered while using antibiotics. $^{25}$

General management strategies: The American Academy of Pediatrics has put guidelines for prescribing antibiotics, it states to prescribe antibiotics in all severe cases of AOM, and in those below 2 years, bilateral and non severe cases. Option is given either to give antibiotics or watchful waiting in those below 2years with unilateral disease or older children. ${ }^{5,8,25}$ Analgesia remains a mainstay in treatment of this condition, but local analgesia adds a little benefit ${ }^{2,5,7,23}$ (Figure 1).

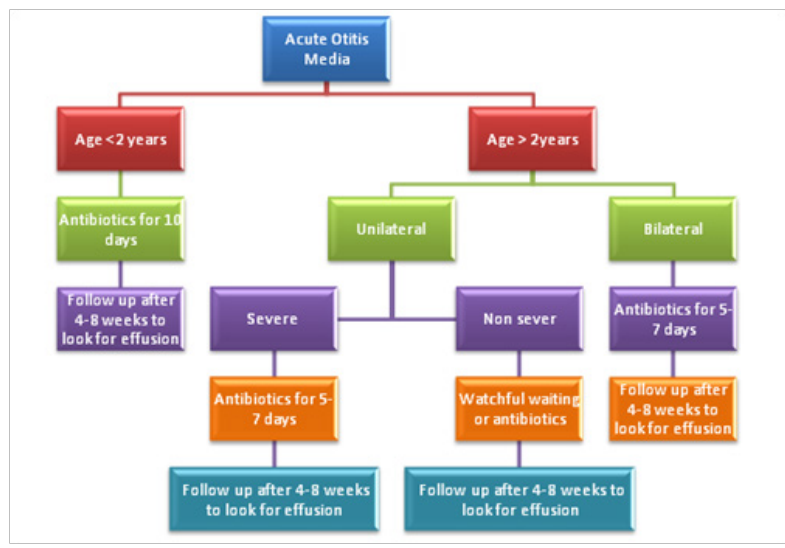

Figure I Management strategies of Acute Otitis Media.

Antibiotic choice: While considering antibiotics amoxicillin remains the first line antibiotic of choice; $;^{5-7}$ as most S. pneumoniae and $\mathrm{H}$. influenza are still susceptible. ${ }^{8}$ If there is a recent history of amoxicillin use in the last 30days, in recurrent cases or if the patient has conjunctivitis then Beta lactamase coverage should be added ${ }^{5,7}$ Cefdinir, cefuroxime and ceftriaxone should be considered in case of penicillin allergy, ${ }^{5}$ Macrolides can be used in allergic cases as well. ${ }^{5,8}$ If severe signs and symptoms persisted more than 2-3days, then antibiotics should be changed to amoxicillin-clavulanate, or second generation or third generation cephalosporin. ${ }^{5}$ The duration of antibiotic treatment is for 10days if below 2 years and 5-7days if above that. ${ }^{5-7}$ It is not indicated to give antihistamine and 
decongestants ${ }^{2,6}$ According to National Institute of Health and Care Excellence- clinical knowledge summaries, physician should proceed with admission in case of children below 3 months with $\mathrm{T}$ above $38^{\circ} \mathrm{C}$, or children between 3 months and 6 months with T above $39^{\circ} \mathrm{C} . .^{5}$

For rOM patients, it is not recommended to give prophylactic antibiotics, ${ }^{5}$ there is controversy about doing grommets insertion in those patients, ${ }^{5}$ but it is found to reduce the recurrence rates,${ }^{6-8}$ and it has also shown to improve quality of life of children in terms of hearing and speech. ${ }^{5}$ No sufficient data is present about using antireflux in $\mathrm{rOM}^{18}$ but some studies found that controlling GERD by anti-reflux medication can decrease rOM and tympanostomy tube insertion rates ${ }^{26}$ (Figure 2).

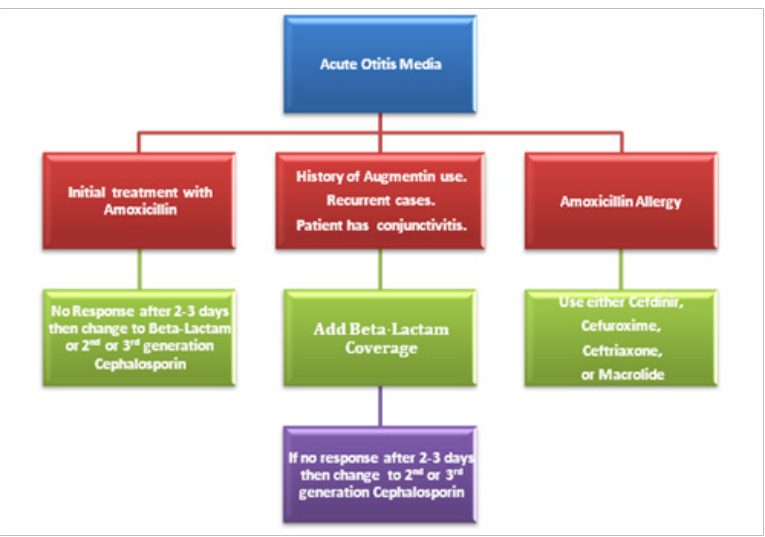

Figure 2 Antibiotherapy for Acute Otitis Media.

Follow up: Effusion will still be evident in $60-70 \%$ of cases after 2 weeks, in $40 \%$ after 4 weeks, and in $25 \%$ after 3 months, ${ }^{7}$ follow up should be done after 4 to 8 weeks for uncomplicated cases of AOM. ${ }^{6}$

\section{Prevention of AOM}

In general $\mathrm{AOM}$ is a symptomatic disease and screening is not indicated. ${ }^{14}$ Breast feeding should be encouraged in the first 6 months and better to continue for the first year. ${ }^{5}$ Smoking avoidance and vaccination are other preventive measures. ${ }^{3,5,7,13}$ PCV7 has been shown to decrease OM rates and tympanostomy tube placement for recurrent diseases $;^{13}$ in fact, this vaccine is more effective if given during infancy. ${ }^{6}$ It can prevent the early episodes of the disease and prevent the mucosal changes, by this it makes the ear less susceptible to infection by other S pneumoniae serotypes not covered by PCV. ${ }^{21}$ Some studies found live attenuated influenza vaccine (LAIV) has decreased the incidence of AOM by $12.4 \%$ if given between $6-71$ months of age, ${ }^{7}$ but others found influenza vaccine is not effective in preventing AOM. ${ }^{6}$ American Academy of Pediatric recommends administration of pneumococcal conjugate Vaccine and influenza vaccine for all children. ${ }^{5}$

Some natural products are used in treatment of AOM; for example, some naturopathic herbal extracts can be used as local ear drops anesthetic agents, ${ }^{13}$ Echinacea pallidum root and Echinacea purpurea have shown some benefit to prevent common cold..$^{13}$ Xylitol is a natural sugar in fruits and found in gums, and found to prevent $\mathrm{OM}$; it works by lowering cpsB (pneumococcal capsular locus) gene expression which causes ultrastructural changes of pneumococcal capsule. ${ }^{13}$ There is conflicting evidence in the role of probiotics in preventing OM. ${ }^{13}$

\section{Conflicts of interest}

Author declares there are no conflicts of interest.

\section{Financial Support}

This research received no specific grant from any funding agency, commercial or not-for-profit sectors.

\section{Acknowledgments}

None.

\section{Funding}

None.

\section{References}

1. Bhutta MF. Epidemiology and pathogenesis of otitis media: construction of aphenotype landscape. Audiol Neurootol. 2014;19(3):210-223.

2. Morris PS, Leach AJ. Acute and chronic otitis media. Pediatr Clin North Am. 2009;56(6):1383-1399.

3. Cunningham M, Guardiani E, Kim HJ, et al. Otitis media. Future Microbiol. 2012;7(6):733-753

4. Pichichero ME. Acute otitis media: Part I. Improving diagnostic accuracy. Am Fam Physician. 2000;61(7):2051-2056.

5. Siddiq S, Grainger J. The diagnosis and management of acute otitis media: American Academy of Pediatrics Guidelines. Arch Dis Child Educ Pract Ed. 2015;100(4):193-197.

6. Wasson JD, Yung MW. Evidence-based management of otitis media: a 5S model approach. J Laryngol Otol. 2015;129(2):112-129.

7. Thomas JP, Berner R, Zahnert T, et al. Acute otitis media--a structured approach. Dtsch Arztebl Int. 2014;111(9):151-159.

8. Atkinson H, Wallis S, Coatesworth AP. Acute otitis media. Postgrad Med. 2015;127(4):386-390

9. Graham Worrall. Acute otitis media. Can Fam Physician 2007;53(12):2147-2148.

10. McCormick DP, Grady JJ, Diego A, et al. Acute otitis media severity: association with cytokine gene polymorphisms and other risk factors. Int J Pediatr Otorhinolaryngol. 2011;75(5):708-712.

11. Blomgren K, Pitkäranta A. Current challenges in diagnosis of acute otitismedia. Int J Pediatr Otorhinolaryngol. 2005;69(3):295-299.

12. Nokso-Koivisto J, Marom T, Chonmaitree T. Importance of viruses in acute otitis media. Curr Opin Pediatr. 2015;27(1):110-115.

13. Levi JR, Brody RM, McKee-Cole K, et al. Complementary and alternative medicine for pediatric otitis media. Int $J$ Pediatr Otorhinolaryngol. 2013;77(6):926-931

14. Schilder AG, Chonmaitree T, Cripps AW, et al. Otitis media. Nat Rev Dis Primers. 2016;2:160-163.

15. Hafrén L, Kentala E, Järvinen TM, et al. Genetic background and the risk of otitis media. Int J Pediatr Otorhinolaryngol. 2012;76(1):41-44.

16. Vergison A. Microbiology of otitis media: a moving target. Vaccine. 2008;26(Suppl 7):G5-G10.

17. Heikkinen T, Chonmaitree T. Importance of respiratory viruses in acute otitis media. Clin Microbiol Rev. 2003;16(2):230-241.

18. Miura MS, Mascaro M, Rosenfeld RM. Association between otitis media and gastroesophageal reflux: a systematic review. Otolaryngol Head Neck Surg. 2012;146(3):345-352.

19. Gould JM, Matz PS. Otitis media. Pediatr Rev. 2010;31(3):102-116.

20. Ngo CC, Massa HM, Thornton RB, et al. Predominant Bacteria Detected from the Middle Ear Fluid of Children Experiencing Otitis Media: A Systematic Review. PLoS One. 2016;11(3):e0150949. 
21. Dagan R, Pelton S, Bakaletz L, et al. Prevention of early episodes of otitismedia by pneumococcal vaccines might reduce progression to complex disease Lancet Infect Dis. 2016;16(4):480-492.

22. Damoiseaux RA, Rovers MM, Van Balen FA, et al. Long-term prognosis of acute otitis media in infancy: determinants of recurrent acute otitis media and persistent middle ear effusion. Fam Pract. 2006;23(1):40-45.

23. Toll EC, Nunez DA. Diagnosis and treatment of acute otitis media: review. JLaryngol Otol. 2012;126(10):976-983.
24. Ryding M, Konradsson K, Kalm O, et al. Auditory consequences of recurrent acute purulent otitis media. Ann Otol Rhinol Laryngol. 2002;111(3 Pt1):261-266.

25. Venekamp RP, Sanders SL, Glasziou PP, et al. Antibiotics for acute otitis media in children. Cochrane Database Syst Rev. 2015;6:CD000219.

26. McCoul ED, Goldstein NA, Koliskor B, et al. A prospective study of the effect of gastroesophageal reflux disease treatment on children with otitis media. Arch Otolaryngol Head Neck Surg. 2011;137(1):35-41. 